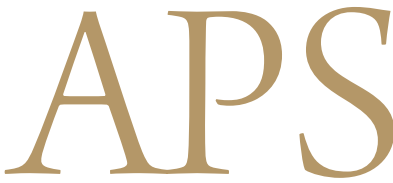

Archives of Plastic Surgery

\title{
Scar Wars: Preferences in Breast Surgery
}

\author{
Cormac W Joyce ${ }^{1}$, Siun Murphy ${ }^{2}$, Stephen Murphy ${ }^{1}$, Jack L Kelly ${ }^{1}$, Colin M Morrison ${ }^{2}$ \\ ${ }^{1}$ Department of Plastic Surgery, University Hospital Galway, Galway; ${ }^{2}$ Department of Plastic Surgery, St Vincent's University Hospital, \\ Dublin, Ireland
}

Background The uptake of breast reconstruction is ever increasing with procedures ranging from implant-based reconstructions to complex free tissue transfer. Little emphasis is placed on scarring when counseling patients yet they remain a significant source of morbidity and litigation. The aim of this study was to examine the scarring preferences of men and women in breast oncoplastic and reconstructive surgery.

Methods Five hundred men and women were asked to fill out a four-page questionnaire in two large Irish centres. They were asked about their opinions on scarring post breast surgery and were also asked to rank the common scarring patterns in wide local excisions, oncoplastic procedures, breast reconstructions as well as donor sites.

Results Fifty-eight percent of those surveyed did not feel scars were important post breast cancer surgery. $61 \%$ said that their partners' opinion of scars were important. The most preferred wide local excision scar was the lower lateral quadrant scar whilst the scars from the deep inferior epigastric artery perforator (DIEP) flap were most favoured. The superior gluteal artery perforator flap had the most preferred donor site while surprisingly, the DIEP had the least favourite donor site.

Conclusions Scars are often overlooked when planning breast surgery yet the extent and position of the scar needs to be outlined to patients and it should play an important role in selecting a breast reconstruction option. This study highlights the need for further evaluation of patients' opinions regarding scar patterns.

Keywords Cicatrix / Breast / Mammaplasty
Correspondence: Cormac W Joyce Department of Plastic Surgery, University Hospital Galway, University Hospital Galway, Newcastle Road,

Galway, Ireland

Tel: +353-91-544-000

Fax: +353-91-544-000

E-mail: cjoyce78@hotmail.com

Received: 18 Mar 2015• Revised: 6 May 2015• Accepted: 26 May 2015

pISSN: 2234-6163 • elSSN: 2234-6171 • http://dx.doi.org/10.5999/aps.2015.42.5.596 • Arch Plast Surg 2015;42:596-600

\section{INTRODUCTION}

In recent times, an increased level of patient education and expectation has led to greater demands on surgeons to produce a better cosmetic outcome following breast cancer surgery. Breast reconstruction is a continually evolving area with a variety of both autologous and implant-based reconstructive options available. The development of perforator-based free flaps has led to a significant increase in the uptake of breast reconstructive surgery, which ranges from 2 to 42 percent in the United States [1] yet the increasing use of complex microvascular procedures has led to issues regarding donor site morbidity and scarring. The increase in the demand for breast reconstruction in the United States is also partly due to the Women's Health and Cancer Rights Act whereby if an insurance company covers a mastectomy, it must also provide cover for the reconstruction.

Mastectomy can have a significantly negative psychological impact [2] and breast reconstruction has been shown to improve body image for women [3]. Body image has been shown to be a key factor in determining differences in quality of life 
when comparing mastectomy and breast conserving therapy [4]. Regardless of the reconstructive technique, the overall patient satisfaction rates are typically high post breast reconstruction [5-7].

A significant factor that can sometimes be overlooked in breast reconstruction is the preference of the individual patient [8]. It has been reported that some surgeons are unaware of their patients concerns and preferences regarding breast reconstruction [8]. Overall, the decision making process should be a joint one between the surgeon and the patient [9] and must be tailormade for each patient individually. The issue of patient preference in breast cancer surgery is relatively underrepresented in the literature and often the decision-making process is led by the surgeon. Interestingly, Lindegren et al. [5] reported that there is a difference between patients' and plastic surgeons' opinions regarding breast reconstruction in the irradiated breast. In their study, surgeons favoured the deep inferior epigastric artery perforator (DIEP) flap whereas the patients were more satisfied with the latissimus dorsi flap reconstruction.

Furthermore, it has also been demonstrated that there is no correlation between the surgical evaluation of the aesthetic outcome in breast reconstruction and overall patient satisfaction [8]. These studies highlight the need for respecting the preference of the patient as it is a critical component of the decisionmaking process and more likely to lead to patient satisfaction, which after all, is the goal of breast reconstruction.

Breast reconstruction following mastectomy leads to various scar patterns depending on the type of reconstruction option chosen. In particular, the donor site scarring is the area associated with most patient dissatisfaction [6]. The morbidity of scarring is well-known and as a consequence, they are a common source of litigation. The scarcity of information in the medical literature relating to scarring in breast surgery is most likely due to the lack of reliable instruments to formally examine scar patterns. The purpose of our study was to explore patient perceptions of scar location in oncological and reconstructive surgery of the breast and to identify factors that may influence their decision-making.

\section{METHODS}

We designed a novel four-page questionnaire to assess patient preferences in relation to scarring following breast reconstructive surgery. It was distributed to 500 participants in two large Irish tertiary referral centres. The participants were a mixture of plastic surgery and breast clinic patients, as well some patients' partners.

As well as focusing on demographics such as age, gender, ethnicity and body mass index (BMI), the first section of the ques- tionnaire focused on the respondents' opinions of scarring postbreast surgery. The questionnaire also asked whether the degree of breast scarring was more important than a good breast shape in clothing postoperatively.

The next three sections of the questionnaire depicted line drawings of breasts with various scar patterns and participants were asked to rank their preference in each category (wide local excision [WLE], oncoplastic and reconstruction). The last section contained line drawings of the donor sites from common reconstructive procedures-DIEP, latissimus dorsi flap (LD), superior gluteal artery perforator (SGAP) flaps and transverse upper gracilis (TUG) flap. Once again, participants were asked to rank these four flap donor sites in order of scar pattern preference.

\section{RESULTS}

A total of 500 people were surveyed in two hospitals and there was even distribution between both sites ( 200 women, 50 men). All surveys were manually entered to Survey Monkey. Most of those surveyed (67\%) were aged between 30 years and 59 years. $78 \%$ of those who responded considered themselves to be average weight while the remaining $22 \%$ described themselves as overweight. $97 \%$ of participants were Caucasian. Of the women surveyed, $22 \%$ had a previous history of breast cancer.

$52 \%$ of respondents felt that a good breast shape in clothing was more important than the degree of breast scarring post-surgery. Overall however, $58 \%$ felt that scars were not important after breast cancer surgery. $62 \%$ of those with a history of breast cancer felt that a good shape in clothes was more important after breast reconstruction than scarring (38\%).

In terms of WLE patterns (Table 1), the most preferred of the four scarring options was the lower lateral quadrant scar, which $55.7 \%$ chose this as their preferred option. The least preferred was the upper medial quadrant scar which $70 \%$ chose as their least favourite choice.

The next section was based on oncoplastic scar patterns and the preferred choice was the circumareolar scar. $72.9 \%$ of respondents choice this as their preferred option whereas $61 \%$ ranked the Wise-pattern scar the least acceptable scar.

Table 1. The wide local excision scar and the percentages of first preferences

Wide local excision pattern Percentage of first preferences

Upper lateral quadrant 21.38

Upper medial quadrant

Lower lateral quadrant

Lower medial quadrant

5.48

55.71

17.43 
Table 2 . The type of reconstruction and the percentages of first preferences

\begin{tabular}{|lc|}
\hline Breast scar & Percentage of first preferences \\
\hline Expander/implant & 31.96 \\
Latissimus dorsi & 1.41 \\
Immediate DIEP & 61.37 \\
Delayed DIEP & 5.26 \\
\hline DIEP, deep inferior epigastric artery perforator. & \\
\hline
\end{tabular}

In terms of breast scars post reconstruction (Table 2), the scar pattern from an immediate DIEP was the highest ranked as $61.4 \%$ had this as their first choice. The least acceptable scars were from the LD flap and delayed DIEP flap ( $45 \%$ and $44 \%$ chose these scar patterns as the least acceptable respectively). For donor site scarring (Table 3), the SGAP was the preferred option as $40 \%$ had this as their first preference. Surprisingly, the DIEP donor site was the least acceptable with $56 \%$ of respondents ranking last.

The results were then filtered to examine separately the results of the males and females surveyed. For women, $58 \%$ felt that a good breast shape in clothing was more important than breast scars (42\%). For the men surveyed, $74 \%$ felt that scarring was more important than the overall shape in clothing (26\%). 65.6\% of the women surveyed thought that scars were not important after breast cancer surgery whilst $72.5 \%$ of men thought scars were important. In relation to partners' opinions of scarring, $53.8 \%$ of the women felt that their partner's opinion was important. $90 \%$ of men felt that their partners opinion of scarring was very important.

The ranking of the line drawings of scarring patterns was almost identical for both men and women. The only difference is that the TUG flap had the preferred donor site for men (47\%) compared to the SGAP flap for women (40\%).

\section{DISCUSSION}

Breast reconstruction has evolved over the years and the surgical options now available reflect these changes. An increased demand for breast reconstruction now exists for a multitude of reasons including increased disease prevalence along with more breast screening, refinement in reconstructive techniques, as a result of legislation as well as an increased public awareness due to high profile celebrities who have undergone breast reconstruction. However, scars are typically the most frequent cause of morbidity and are traditionally associated with a high risk of litigation. The purpose of this study was to identify patient preferences in relation to scar location as well as to ascertain the factors involved in the decision-making process.
Table 3 . The donor sites and the percentages of first preferences

\begin{tabular}{lc|}
\hline Donor site & Percentage of first preferences \\
\hline DIEP & 16.87 \\
Latissimus dorsi & 16.97 \\
SGAP & 39.98 \\
TUG & 26.18 \\
\hline DIEP, deep inferior epigastric artery perforator; SGAP, superior gluteal artery \\
perforator; TUG, transverse upper gracilis.
\end{tabular}

The choice of the most suitable reconstruction option is not a decision that should be made lightly and requires a great deal of discussion between the surgeon and the patient [10]. This communication has been shown to sometimes be deficient and this can have a long-term adverse outcome [7,11]. In fact, it has been reported that disparities exist between patients' preferences and surgeons' preferences and this can lead to lower patient satisfaction rates or even regret after surgery $[12,13]$. In our study, the majority of those (56\%) surveyed preferred the WLE scar in the lower outer quadrant of the breast. This area tends to be the most hidden area of the breast in clothing and would also be the least noticeable position on the breast for the patient. In contrast, the least preferred area for WLE scarring is the upper inner quadrant as this scarring would be quite noticeable in any clothing that revealed cleavage. This echoes the findings made by Coutinho et al. [14] in their paper from 2001. Not only is the upper inner quadrant felt by women to be the least preferable aspect of the breast in terms for scarring but it is also felt to be the most important portion of breast contour and shape [15]. By far the most preferred oncoplastic scar pattern was the circumareolar scar whilst the least acceptable was the scar from the Wise-pattern incision. Not surprisingly, this indicates that those surveyed preferred the minimal amount of scarring possible. The DIEP is often considered the gold standard for breast reconstruction by many surgeons [5] for its aesthetic result and natural ptosis. However, it has been reported that surgeons may overestimate the result of a technically more advanced reconstruction [5]. It is not surprising that the immediate DIEP reconstruction pattern was the most preferred option by both men and women. However, the DIEP abdominal donor site was the least preferred of the four donor sites and this is likely due to the fact that those surveyed were only asked to rank scar patterns and not overall outcome. The DIEP donor site scar is the most extensive scar and was therefore chosen as the least preferred option. The added benefit of removing excess abdominal tissue for DIEP reconstruction was not made known to respondents and this may well have skewed these results. Those surveyed were also not made aware of the complications and vari- 
ous donor site morbidities associated with each reconstruction option and this certainly would have affected the results too. In particular, the SGAP donor site was the most preferred donor site yet those surveyed were not aware that the SGAP flap frequently results in significant buttock asymmetry and can have an uncomfortable donor site. In a study by Yueh et al. [16], patient satisfaction post breast reconstruction was assessed and a comparative evaluation of implant-based techniques, $\mathrm{LD}$ and DIEP flaps was performed. They found that the highest postoperative satisfaction rates were with the DIEP flap and the lowest were with the implant-based reconstruction.

Very little information can be found in the medical literature concerning patient preferences on scarring in breast surgery. Lindegren et al. [5] described how patients were happier with the LD donor site than the DIEP donor site. This may be due to a greater morbidity encountered at the donor site with DIEP patients and the fact that the $\mathrm{LD}$ scar is not readily visible by the patients. In our study, the DIEP donor site was the least favoured donor site whilst the SGAP donor site was the most preferred. This indicates that patients seemed to favour scar patterns that were not readily visible by the patient. However, if the added benefit of the abdominoplasty effect of the DIEP flap was known to those surveyed, the results may have been quite different. Equally, if the morbidities associated with the DIEP donor site were known to those surveyed, it may have discouraged them from choosing this option.

To our knowledge, this is the first study of its kind that surveyed men about their opinions on scarring in breast surgery. Overall, the answers of the men and women questioned were very similar in all parts of the survey. More than half the women surveyed felt that their partner's opinions were very important in relation to scarring yet almost all of the men surveyed felt that their partners opinion on scarring was highly important. This highlights the fact that partners should attend breast reconstruction consultations and should be involved in the decision-making process. This is an important issue that should be further examined as it is common for women to attend consultations alone. As clinicians we should encourage partners to attend and involve them in the consultation.

Because scarring can vary greatly between different individuals and ethnicities we chose to use line diagrams depicting scars instead of photographs for our questionnaire. We focused on preferences of scar positioning rather than other features of scars such as pigmentation, height, tenderness and pliability. Despite the lack of realism, we felt line drawings were more easily standardized and respondents were more likely to focus solely on the scar in the diagrams than if photographs were used. Despite these drawings being a limitation of this type of study, we feel that they were better suited to the questionnaire than the use of photographs. As scars vary from person to person, it would have been impossible to standardize the photographs. If photographs were to be used in a study like this, a significant number would be required in order to demonstrate a large variety of breast aesthetic characteristics such as symmetry, size, ptosis and projection as well as a variety of scarring types and pigmentation $[17,18]$.

Limitations do exist in a study of this nature. Breast cancer patients only constituted $22 \%$ of the 400 women surveyed. Surprisingly, there was no difference in the scarring preferences between women who had breast cancer and those who had not. Despite this fact, it would have been ideal to have a larger cohort of breast cancer patients to assess with regards to scarring preferences. We did not ask those with a history of breast cancer whether they had undergone reconstructive surgery in the past. It would be worth repeating this study to compare scarring preferences between preoperative and postoperative breast reconstruction patients.

The plastic surgery literature is awash with articles pertaining to breast reconstruction yet there is a dearth of information regarding donor site scarring. However, in breast surgery, a fine balance must be maintained between the amount of scarring and the overall shape of the breast. The extent and position of the scar needs to be outlined to patients prior to surgery and it should play an important role in selecting a breast reconstruction option. This study highlights the need for further evaluation of patients' opinions regarding scar patterns.

\section{REFERENCES}

1. Matros E, Yueh JH, Bar-Meir ED, et al. Sociodemographics, referral patterns, and Internet use for decision-making in microsurgical breast reconstruction. Plast Reconstr Surg 2010; 125:1087-94.

2. Manderson L, Stirling L. The absent breast: speaking of the mastectomied body. Fem Psychol 2007; 17:75-92.

3. Cocquyt VF, Blondeel PN, Depypere HT, et al. Better cosmetic results and comparable quality of life after skin-sparing mastectomy and immediate autologous breast reconstruction compared to breast conservative treatment. $\mathrm{Br} \mathrm{J}$ Plast Surg 2003;56:462-70.

4. Bartelink H, van Dam F, van Dongen J. Psychological effects of breast conserving therapy in comparison with radical mastectomy. Int J Radiat Oncol Biol Phys 1985; 11:381-5.

5. Lindegren A, Halle M, Docherty Skogh AC, et al. Postmastectomy breast reconstruction in the irradiated breast: a comparative study of DIEP and latissimus dorsi flap outcome. Plast Reconstr Surg 2012;130:10-8. 
6. Gilboa D, Borenstein A, Floro S, et al. Emotional and psychosocial adjustment of women to breast reconstruction and detection of subgroups at risk for psychological morbidity. Ann Plast Surg 1990;25:397-401.

7. Abu-Nab Z, Grunfeld EA. Satisfaction with outcome and attitudes towards scarring among women undergoing breast reconstructive surgery. Patient Educ Couns 2007;66:243-9.

8. Lee CN, Hultman CS, Sepucha K. What are patients' goals and concerns about breast reconstruction after mastectomy? Ann Plast Surg 2010;64:567-9.

9. Joyce CW, Morrison CM, Sgarzani R, et al. Patient preferences in an online breast reconstruction resource. J Plast Reconstr Aesthet Surg 2013;66:e380-1.

10. Gopie JP, Hilhorst MT, Kleijne A, et al. Women's motives to opt for either implant or DIEP-flap breast reconstruction. J Plast Reconstr Aesthet Surg 2011;64:1062-7.

11. Alderman AK, Hawley ST, Waljee J, et al. Understanding the impact of breast reconstruction on the surgical decisionmaking process for breast cancer. Cancer 2008;112:489-94.

12. Rolnick SJ, Altschuler A, Nekhlyudov L, et al. What women wish they knew before prophylactic mastectomy. Cancer Nurs 2007;30:285-91.
13. Sheehan J, Sherman KA, Lam T, et al. Association of information satisfaction, psychological distress and monitoring coping style with post-decision regret following breast reconstruction. Psychooncology 2007;16:342-51.

14. Coutinho M, Southern S, Ramakrishnan V, et al. The aesthetic implication of scar position in breast reconstruction. Br J Plast Surg 2001;54:326-30.

15. Bailey SH, Saint-Cyr M, Oni G, et al. Aesthetic subunit of the breast: an analysis of women's preference and clinical implications. Ann Plast Surg 2012;68:240-5.

16. Yueh JH, Slavin SA, Adesiyun T, et al. Patient satisfaction in postmastectomy breast reconstruction: a comparative evaluation of DIEP, TRAM, latissimus flap, and implant techniques. Plast Reconstr Surg 2010;125:1585-95.

17. Kim MS, Rodney WN, Reece GP, et al. Quantifying the aesthetic outcomes of breast cancer treatment: assessment of surgical scars from clinical photographs. J Eval Clin Pract 2011;17:1075-82.

18. Brolmann FE, Eskes AM, van de Kar AL, et al. Are digital photographs reliable to assess donor site scars? An intermethod analysis and validity testing. J Plast Reconstr Aesthet Surg 2013;66:1632-5. 